\title{
EVALUACIÓN Y PONDERACIÓN DE LA PARTICIPACIÓN CIUDADANA EN EL URBANISMO \\ Sistema de evaluación de los procesos de participación, su impacto y evolución temporal
}

\author{
Evaluation and weighting of urban participation \\ System for evaluating of participation processes, their impact and time evolution
}

Moreno Balboa, Carmen

Departamento de Urbanística y Ordenación del Territorio, ETSAM. Universidad Politécnica de Madrid.

balboamorenocarmen@gmail.com

\section{RESUMEN}

La participación ciudadana en la construcción de la ciudad es un elemento fundamental para el avance social y desarrollo democrático. Los procesos son numerosos, sin embargo, a diferencia de otras políticas públicas, no existe un sistema de evaluación que permita conocer los casos de éxito y extraer conclusiones útiles y replicables en otros procesos. Las experiencias urbanas son únicas, el software urbano constituido por sus comunidades y las relaciones y redes creadas entre éstos son únicas y no aplicables a otros entornos; la evaluación nos permite extraer un juicio de valor fundamentado y unas conclusiones para nuevos procesos. El sistema de evaluación propuesto, mediante el cumplimiento de una matriz, es sencillo y universal, permite una evaluación contrastada en el tiempo, es una herramienta sencilla y fácilmente implementable en los procesos abiertos, y los ya concluidos. Es momento de abordar la participación de modo científico y estructurado, es momento de evaluar.

Palabras clave: participación ciudadana, urbanismo colaborativo, gobernanza, evaluación.

Bloque temático: Regeneración urbano-habitacional.

\begin{abstract}
The citizens participation and their collaboration in the city's construction is a fundamental element for the social progress and for the democratic development. There are numerous procedures but, unlike other public politics, there is no evaluation system that provides information about the successful cases in order to develop helpful conclusions to get better results in future projects. The urban experiences are unique as well as the urban software formed by the communities and the relationships in between them. This evaluation helps us to find and extract new and fundamented conclusions for future procedures. The evaluation system we propose is universal and simple, which allows an evaluation contrasted through time. It's a simple and easy to use tool for open procedures as well as for finished ones. It's time to approach participation in a scientific and structured way, it's time to evaluate.
\end{abstract}

Keywords: citizen participation, collaborative urbanism, governance, evaluation Topic: Urban-housing regeneration 


\section{Introducción}

La participación ciudadana está en constante evolución, cada vez más presente, tomando nuevas formas, metodologías, herramientas... en todos los procesos urbanos desde la toma de contacto, el análisis y diagnóstico de la ciudad existente hasta las fases de planificación e incluso de ejecución. A todas las escalas, desde planeamiento de amplios desarrollos de ciudad o del territorio, hasta pequeñas intervenciones de acupuntura urbana. En todos los ámbitos que abarca el hecho urbano: sociales, económicos, educativos, presupuestarios, ambiente y calidad urbana, convivencia, movilidad, salud, ...

Sin embargo, no consta que de dichos procesos se hayan realizado evaluaciones sistemáticas, tanto de la situación de partida, como del proceso y de sus resultados o de su evolución en el tiempo. De hecho ni siquiera existe un consenso sobre cuáles son los parámetros o indicadores que podrían facilitar dicha evaluación. Ejemplos como el de la "Guía Práctica, Evaluación de Procesos Participativos" de los Observatorios Locales de Democracia Participativa del Ayuntamiento de Barcelona aportan herramientas y criterios de evaluación incluso sistematizado en tablas más o menos sencillas pero no ha sido verificada o suficientemente difundida para su calibración efectiva.

En un momento como el actual, en el que son tantas las iniciativas de participación, contar con una herramienta de evaluación simplificaría los procedimientos. Al menos, para no repetir lo que ya se ha comprobado que no funciona, y aprovechar lo que sí ha funcionado, y seguir perfeccionando, avanzando, aprendiendo. Los procesos abiertos proporcionan información sobre la utilidad de éstos, lo que facilita mantenerlos vivos -pues uno de los principales motivos por los que desfallecen, es la pérdida de la motivación y la constatación de la ausencia de resultados, y por tanto de la inutilidad del esfuerzo realizado, por parte de los participantes.

Cada ciudad es diferente, lo que en una funciona no sirve para la siguiente, cada caso es único. Los profesionales del urbanismo lo saben, pero hay datos, elementos, procesos, planteamientos, resultados que sí es posible analizar y de los que se podrían extraer ciertos criterios y parámetros que sean replicables, pautas de funcionamiento deducidas a partir de la praxis, el saber obtenido por el método heurístico, de la prueba y el error. Aprendizaje que ayuda a simplificar, mejorar y crear procesos más eficientes, evitando los errores ya cometidos.

\section{Objetivos}

Cada vez son más numerosas las iniciativas ciudadanas, cívicas, cuyo objetivo, de algún modo, es la mejora de la calidad de vida en las ciudades, como vemos en el "Atlas de Iniciativas Vecinales", iniciado en 2015 y que continua su proceso de actualización y especialización, los campos de actividad son innumerables: desarrollo comunitario, arte urbano, cultura libre, igualdad ecología, consumo, otras economías, educación expandida, feminismos, ...

En la relación entre dichas iniciativas y la administración pública se observan cambios, aunque la cuestión planteada por Rosario del Caz y Manuel Sarabia (2005) sigue sin una respuesta convincente: “¿No es cierto que ninguna comunidad o ciudad (en opinión de Janice E. Perlman) "puede realizar a la larga su completo potencial sin una vibrante sociedad civil independiente y sin la participación activa de su ciudadanía (dos aspectos complementarios, pero no sinónimos)?". 


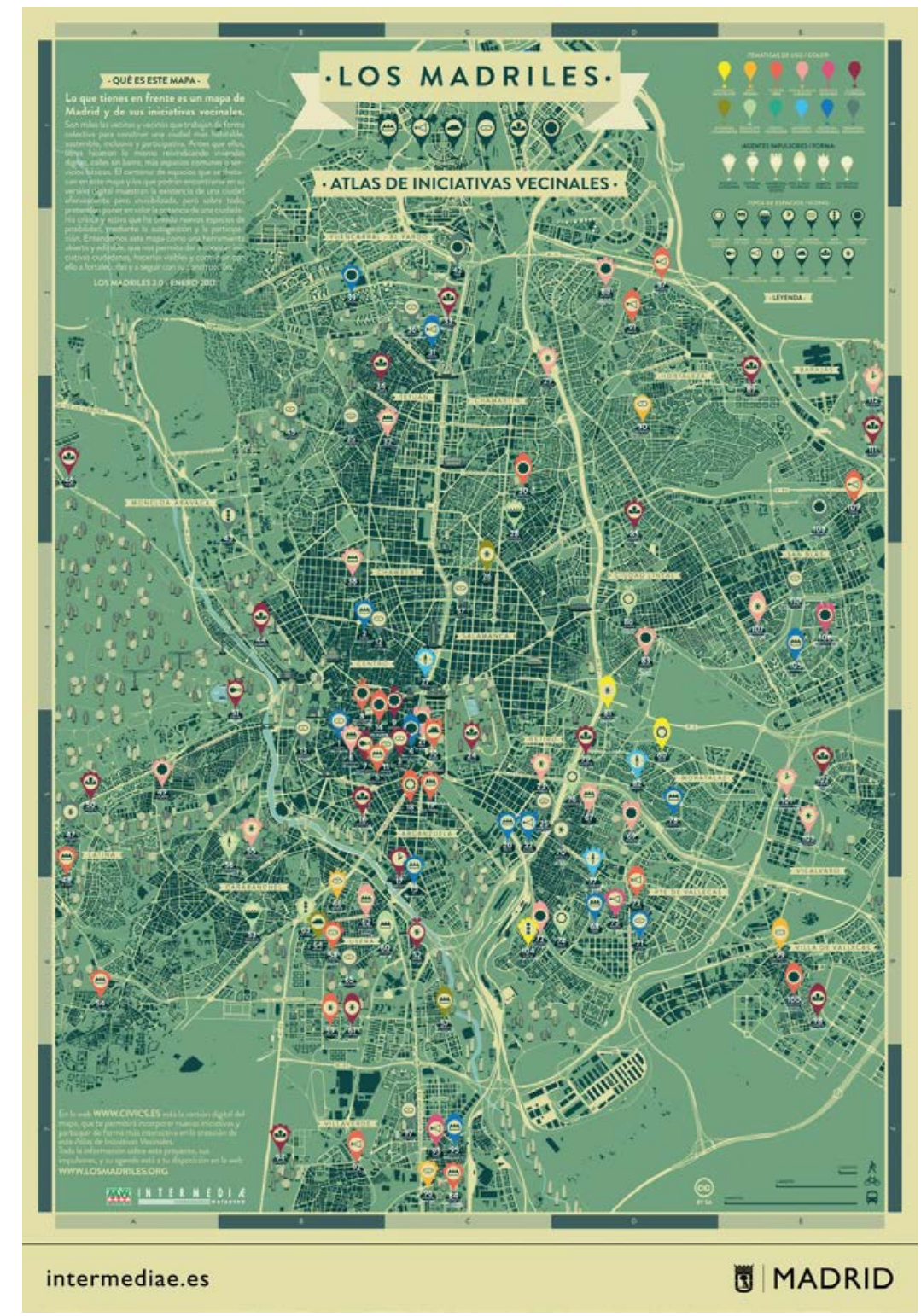

Figura 1. Mapa Los Madriles. Madrid V2.0. 2017

En la gestión de la ciudad es indudable que es la administración quien debe asumir el impulso y fomento de la actividad urbanística, parte de ésta es la participación ciudadana como invita la iniciativa de Los Madriles: "REFLEXIÓN Y AUTOCRÍTICA para autoridades y responsables locales. Este mapa quiere invitar a pensar nuevas formas de interlocución con la ciudadanía que ayuden a mejorar los barrios y distritos de la ciudad, tomando las decisiones de forma conjunta con sus vecinos. Una invitación a pensar entre todos cómo construir la ciudad de forma colectiva y participativa."

En estos últimos años asistimos a nuevos intentos, pruebas, iniciativas como las del Ayuntamiento de Madrid de las que habla José Fariña en su artículo "Once plazas de Madrid [+1]": "Es bien conocida la inutilidad del sistema de participación en el planeamiento urbanístico español (y en todas las Comunidades Autónomas), legalmente obligatorio pero inoperante desde el punto de vista práctico. Es por eso que iniciativas como la del Ayuntamiento de Madrid tienen un gran interés y hay que analizarlas sin perjuicios para ver si suponen realmente un avance sobre lo experimentado hasta el momento en el campo urbanístico". 
Potenciar dichas iniciativas parece imprescindible, como apunta Fariña; analizar los procesos y extraer conclusiones prácticas permite avanzar en esta colaboración entre administraciones y ciudadanos, en este sentido desarrollar un método de evaluación es una herramienta necesaria.

De hecho, la evaluación de políticas públicas es una práctica habitual en otros ámbitos de la actividad política:

"La evaluación, en el actual contexto político, aparece ligada a aspectos sociales y de transparencia democrática, en estrecha relación con la publicidad de sus informes y la participación plural de los actores que entran en juego". (AEVAL, 2010:4)

"La evaluación no es sólo una comprobación de conformidad de la acción pública, sino que expresa un juicio de valor sobre el éxito de los resultados y los impactos -deseados o no- de las políticas públicas y ese juicio de valor se debe extraer con el mayor rigor y garantías, de ahí la exigencia de una sólida metodología". (AEVAL, 2010:4)

Aprendiendo de la evaluación de las políticas públicas que se viene desarrollando desde 2007, podemos constatar que en el ámbito del urbanismo no ha habido avances significativos, baste comprobar a modo de ejemplo sobre qué Buenas Prácticas han recaído los Premios a la Calidad e Innovación en la Gestión Pública en sus casi 10 años de existencia, principalmente en políticas económicas y de gestión interna.

Pueden ser varias las causas de esta realidad, pero a los efectos del actual estudio nos centraremos en dos fundamentales dada la complejidad de la materia: las fases de la construcción de la ciudad en la que se produce la actividad participativa y el método utilizado. Ambos aspectos se desarrollan en el apartado siguiente.

En cuanto la relevancia de la participación en el urbanismo, interesa la reflexión de Fariña sobre lo que debería ser: "Participar no es decidir sin tener ni idea de qué se está decidiendo. Participar en urbanismo no es un acto único como el de votar a nuestros representantes políticos. No es decidir entre un proyecto $u$ otro ya terminados. Participar debería ser un proceso largo, complejo, en el que se produce una interacción entre los técnicos, los políticos y la sociedad a lo largo de todo el desarrollo del proyecto, de resultas del cual hay un acercamiento de posturas sobre cómo queremos que, en realidad, sea el ámbito físico de nuestras vidas". De hecho estamos hablando de producir cambios culturales y sociales que únicamente se pueden alcanzar con políticas públicas que se mantengan en el tiempo. No se trata únicamente de procesos puntuales y evaluaciones cuantitativas: el número de participantes, de propuestas viables o cambios en un plan; sino de la transformación de las prácticas y procedimientos administrativos y de gestión pública, incorporando la participación pública, y evaluando en el medio y largo plazo los cambios e impactos culturales y sociales que se producen. La Carta Europea de la Democracia Participativa en Procesos Urbanísticos apunta en esta dirección: "La cultura debe ser considerada como el cuarto pilar de la sostenibilidad. Por lo tanto, tenemos que recordar que no hay acción urbanística que no conlleve implicaciones culturales. La información, la formación y la participación son factores culturales estrechamente relacionados".

Estos cambios están ampliamente estudiados en las teorías del cambio (MOORE, 1998), y el modelo lógico para producir y evaluar la eficiencia de programas y políticas del sector público y organizaciones sin ánimo de lucro, en las que el objetivo final no es un beneficio económico sino cambios sociales, culturales, educativos... 
ACTIVIDADES

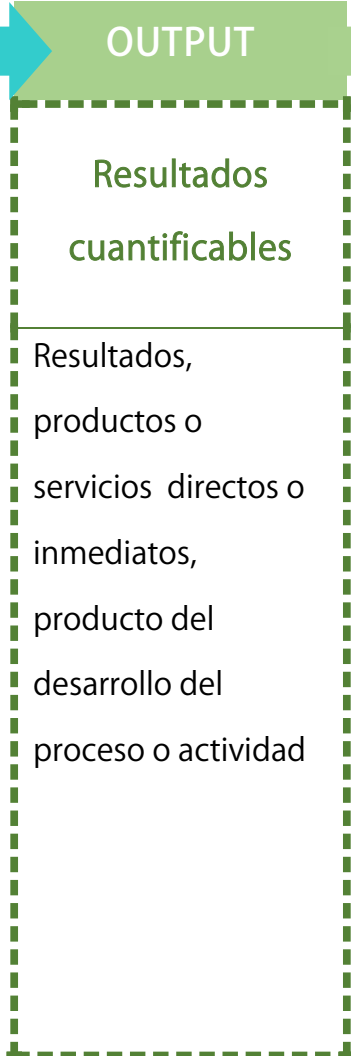

\section{IMPACTO}

OUTCOME

Cambios no

cuantificables

Efectos probables o alcanzados a corto y medio plazo de los resultados de una intervención $\quad 0$ proceso.

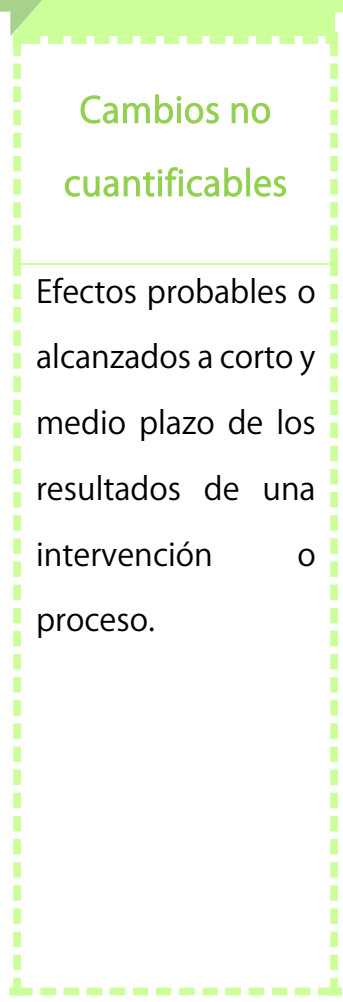

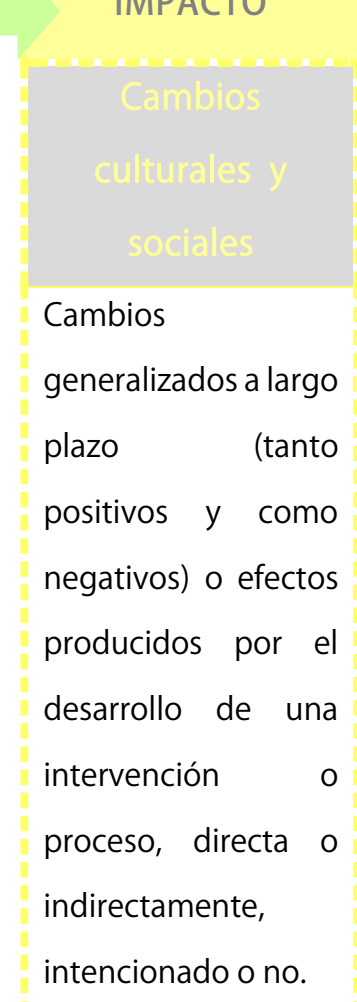

Tabla 1. Modelo lógico de la Teoría del Cambio. Fuente: Elaboración propia

El éxito de los procesos de participación se centra en la construcción del consenso, como alternativa al método tradicional decisional de arriba a abajo. Las características de los procesos colaborativos, según Healey.(20061997), Innes y Booher (2000), son los siguientes:

- Implicación de múltiples actores en nuevas áreas de diálogo a las que acuden libremente y crean sus normas de comportamiento.

- Tarea que les motive y sea significativa, con la perspectiva de producir resultados.

- Reconocen las dependencias mutuas

- Todos tienen el mismo poder y están predispuestos a escuchar y respetar al resto de los participantes.

- Cuentan con la misma información accesible y compartida.

- Los actores deben ser representativos.

- Los valores de justicia inclusiva y sostenibilidad tienen que ser vigentes en todo el proceso.

- Se comprometen para llegar al consenso. Consensus building: construcción del consenso (como alternativa al enfoque de arriba-abajo, regla de la mayoría o el enfrentamiento)

- Se integra la experiencia y el conocimiento del lugar y el conocimiento científico y técnico.

\section{Resultados / Beneficios:}

- Se genera conocimiento y poder a través del aprendizaje colectivo y la capacidad de innovar.

- Se opera un cambio en los participantes: nuevas ideas, otras visiones.

- Su mantenimiento crea marcos de referencia y se pueden acordar intervenciones.

- El proceso puede dar lugar a combinar innovación y eficacia.

- Generación de red: relaciones personales e institucionales, fundamentadas en el entendimiento y la confianza mutua: relational resources, que derivan en capital intelectual y social. Nueva forma de poder: búsqueda de soluciones compartidas y flujo de información: distributed self-organizing action. 
- Construcción de un alto grado de confianza.

- Mayor capacidad de resolver los problemas que otras formas de política.

- Mayor capacidad institucional: capital social, intelectual y político. Los participantes son más competentes, están mejor informados y confían más en su capacidad para cambiar las cosas.

- Enriquece el resultado: capacidad de aprendizaje, interacción, retroalimentación y combinación de ensayo y error.

- Resultado con mayor nivel de complejidad, productividad, competencia, estabilidad, eficacia y organización.

Este mismo planteamiento lo encontramos en el Plan de Acción de la Agenda Urbana Española, como una de las acciones prioritarias para abordar en el corto plazo por parte de la Administración General del Estado, en el apartado 3.4 Fomentar la transparencia de las administraciones y la participación ciudadana basada en el conocimiento previo, la educación y la formación. ${ }^{1}$

En definitiva, el objetivo último del presente trabajo de investigación es el fomento de la participación ciudadana, extraer con el mayor rigor, un juicio de valor sobre los aspectos más destacados de los procedimientos, los resultados y el impacto de los procesos participativos, con el fin de asegurar el impacto social positivo que generan.

\section{Metodología}

De las prácticas realizadas y el estado del arte metodológico, es posible extraer una serie de categorías de evaluación que sirvan para desarrollar un sistema de medición y análisis de los procesos de participación, tanto de los ya finalizados y como de las futuras iniciativas, que contribuya a su mejora continua. El conocimiento individual contrastado y compartido puede ayudar a la mejora de este tipo de experiencias, y contribuir a su empleo generalizado con mayor garantía de efectividad.

Partiendo de la racionalización de los procesos de participación, se han identificado sus componentes, características y parámetros diferenciadores en sus principales fases, a partir de éstas se ha confeccionado una matriz de evaluación y ponderación de los procesos que permite, además, monitorizar su evolución en el tiempo. Para ello se ha tomado como base de referencia el trabajo del Consejo de Europa 2009, que incorpora en la página siguiente. En ésta intervienen los dos aspectos considerados anteriomente: la fase de ejecución de la ciudad y el método utilizado en el proceso participativo.

El interés de esta matriz se basa en que plantea los mejores procesos o mecanismos para la participación, en función de los niveles de ésta, desde el nivel inferior -información- pasando por la consulta y el diálogo hasta el nivel superior de Alianzas, de modo cruzado con las decisiones o resultados que se pueden esperar del proceso participativo en cada nivel.

Mediante un amplio trabajo estudio de las características, variables y parámetros de los procesos de participación y el análisis de cinco casos, se ha generado una matriz de evaluación de la participación que analiza el proceso, los resultados y el impacto producido. Con ello se puede parametrizar y evaluar las experiencias de participación, permitiendo su comparación y la extracción de conclusiones fiables y juicios de valor con mayor rigor.

Además, se plantea un sistema de valoración que facilita el análisis cualitativo de las experiencias, el seguimiento de su desarrollo en el tiempo y comparación con otros procesos. Por tanto el sistema permite una valoración cuantitativa en consonancia con la paramétrica.

\footnotetext{
${ }^{1}$ AUE [2019] Ministerio de Fomento. "No obstante, la participación ciudadana no será real ni efectiva mientras que no exista una verdadera conciencia que genere compromisos en relación con los intereses generales que conforman y confluyen en las ciudades y en los entornos urbanos. Para ello la formación y la difusión de los objetivos de la Agenda Urbana son claves".
} 


\section{VALORACIÓN}

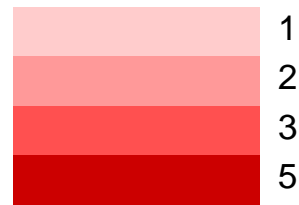

Figura 2. Gradación y valoración de procesos participativos. Fuente: Elaboración propia

\begin{tabular}{|c|c|c|c|c|c|c|}
\hline $\begin{array}{c}\text { Toma de } \\
\text { decisiones }\end{array}$ & $\begin{array}{c}\text { Elaboración de } \\
\text { programas }\end{array}$ & Formulación & Decisión & Aplicación & Supervisión & $\begin{array}{l}\text { Reformula- } \\
\text { ción }\end{array}$ \\
\hline Alianzas & $\begin{array}{c}\text { Grupo de trabajo o } \\
\text { comité }\end{array}$ & Formulación conjunta & $\begin{array}{l}\text { Toma conjunta } \\
\text { de decisiones } \\
\text { Decisión conjunta }\end{array}$ & Alianzas estratégicas & $\begin{array}{l}\text { Grupos de } \\
\text { trabajo o } \\
\text { comité }\end{array}$ & $\begin{array}{l}\text { Grupos de } \\
\text { trabajo o } \\
\text { comité }\end{array}$ \\
\hline Diálogo & $\begin{array}{c}\text { Audiencias y foros } \\
\text { públicos } \\
\text { Foros de } \\
\text { ciudadanos y } \\
\text { consejos futuros } \\
\text { Interlocutor } \\
\text { gubernamental clave }\end{array}$ & $\begin{array}{c}\text { Audiencias y paneles } \\
\text { de preguntas y } \\
\text { respuestas } \\
\text { Seminarios de } \\
\text { expertos } \\
\text { Comités de múltiples } \\
\text { partes interesadas y } \\
\text { órganos consultivos }\end{array}$ & $\begin{array}{l}\text { Sesiones } \\
\text { plenarias o } \\
\text { reuniones de } \\
\text { comités de } \\
\text { participación } \\
\text { libre }\end{array}$ & $\begin{array}{l}\text { Seminarios de } \\
\text { creación de } \\
\text { capacidad } \\
\text { Seminarios de } \\
\text { formación }\end{array}$ & $\begin{array}{l}\text { Grupos de } \\
\text { trabajo o } \\
\text { comité }\end{array}$ & $\begin{array}{l}\text { Seminarios y } \\
\text { foros de } \\
\text { deliberación }\end{array}$ \\
\hline Consulta & $\begin{array}{c}\text { Presentación de } \\
\text { solicitudes } \\
\text { Consulta en línea u } \\
\text { otras técnicas }\end{array}$ & $\begin{array}{l}\text { Audiencias y paneles } \\
\text { de preguntas y } \\
\text { respuestas } \\
\text { Seminarios de } \\
\text { expertos } \\
\text { Comités de múltiples } \\
\text { partes interesadas y } \\
\text { órganos consultivos }\end{array}$ & $\begin{array}{l}\text { Sesiones } \\
\text { plenarias o } \\
\text { reuniones de } \\
\text { comités de } \\
\text { participación } \\
\text { libre }\end{array}$ & $\begin{array}{l}\text { Acontecimientos, } \\
\text { conferencias, } \\
\text { foros, seminarios }\end{array}$ & $\begin{array}{l}\text { Mecanismos } \\
\text { de } \\
\text { intercambio } \\
\text { de opiniones }\end{array}$ & $\begin{array}{l}\text { Conferencias } \\
\text { o reuniones } \\
\text { Consulta en } \\
\text { línea }\end{array}$ \\
\hline $\begin{array}{l}\text { Informa- } \\
\text { ción }\end{array}$ & $\begin{array}{c}\text { Acceso fácil y abierto } \\
\text { a la información } \\
\text { Investigación } \\
\text { Realización de } \\
\text { campañas y presión } \\
\text { Sitio Web con } \\
\text { documentos clave }\end{array}$ & $\begin{array}{c}\text { Acceso abierto y } \\
\text { libre a documentos } \\
\text { de política } \\
\text { Web con documentos } \\
\text { clave } \\
\text { Realización de } \\
\text { campañas y presión } \\
\text { Difusión a través de la } \\
\text { Web } \\
\text { Aportaciones de } \\
\text { las investigaciones }\end{array}$ & $\begin{array}{l}\text { Realización de } \\
\text { campañas y } \\
\text { presión }\end{array}$ & $\begin{array}{l}\text { Acceso abierto a } \\
\text { la información } \\
\text { Sitio Web para } \\
\text { acceder a la } \\
\text { información } \\
\text { Alertas correo e. } \\
\text { Preguntas } \\
\text { frecuentes } \\
\text { Anuncios públicos } \\
\text { de procesos de } \\
\text { licitaciones }\end{array}$ & $\begin{array}{l}\text { Acceso } \\
\text { abierto a la } \\
\text { información } \\
\text { Recopilación } \\
\text { de pruebas } \\
\text { Evaluacione } \\
\text { s } \\
\text { Estudios de } \\
\text { investiga- } \\
\text { ción }\end{array}$ & $\begin{array}{c}\text { Acceso abierto } \\
\text { a la } \\
\text { información }\end{array}$ \\
\hline
\end{tabular}

Tabla 2. Matriz: Niveles de participación y proceso de toma de decisiones. Fuente: Código Consejo de Europa 2009 


\subsection{Estructura y parámetros utilizados}

El sistema de evaluación que se presenta se basa en dos principios fundamentales: simplicidad y universalidad. Se busca la simplicidad en el convencimiento que de que es garantía de uso, por cualquier interesado sin necesidad de grandes conocimientos sobre la materia o sobre procedimientos de evaluación pues los datos que se manejan pueden ser de conocimiento general y de aplicación directa. La universalidad se refiere, en este caso, a la posibilidad de aplicar el sistema a todo tipo de procesos, sin necesidad de adaptaciones específicas o crear un elenco de matrices y tablas en función del tipo u objetivo de la actividad de participación evaluada.

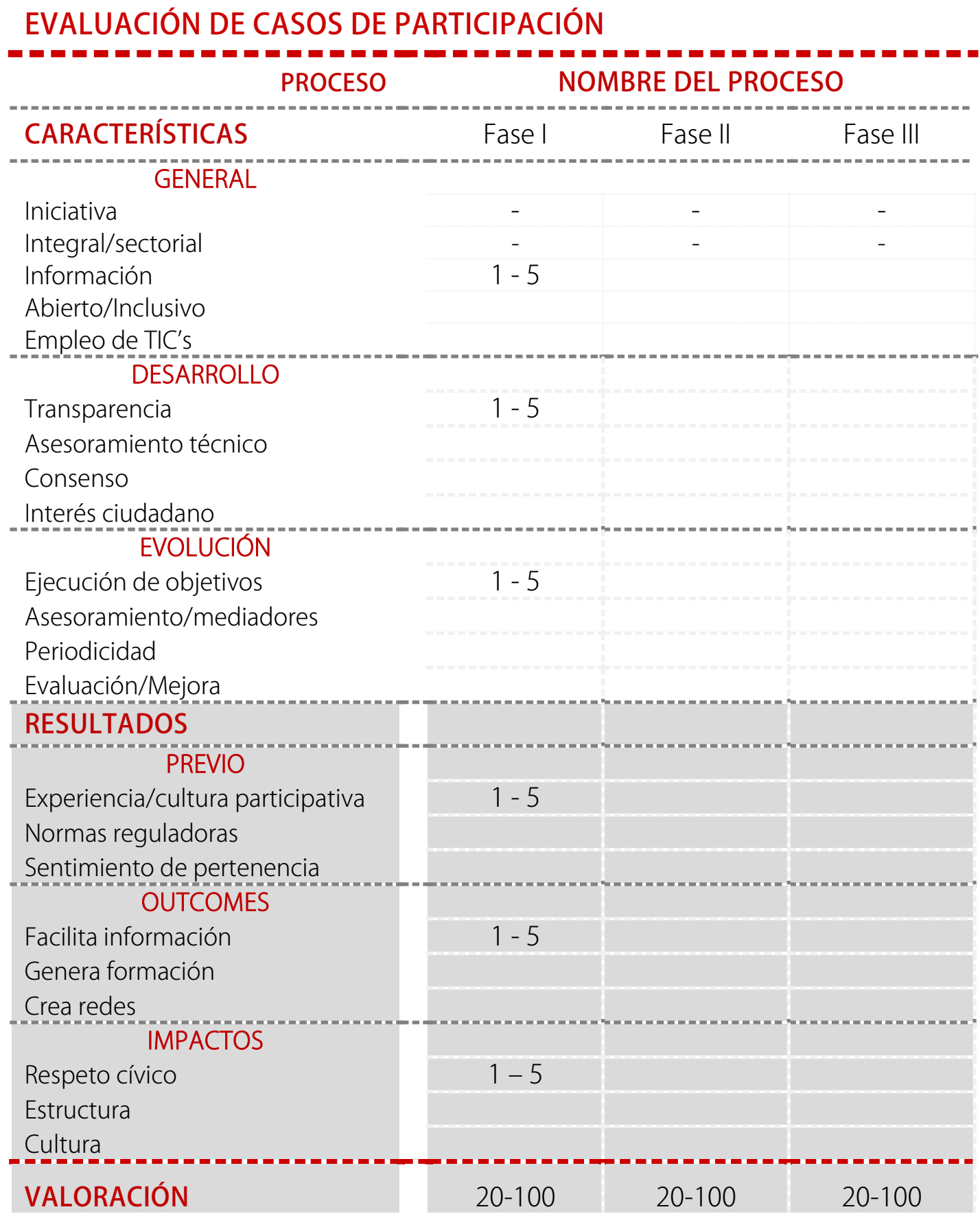

Tabla 3. Evaluación y seguimiento de procesos de participación. Fuente: Elaboración propia 
Además se busca obtener los suficientes datos del proceso para facilitar un análisis integral tanto del proceso en sí mismo como su seguimiento en el tiempo, que se completa con el sistema de valoración cuantitativa que, aunque algo subjetivo, ofrece un valor final fácilmente manejable y del que obtener conclusiones reales de la acción.

La matriz de evaluación se estructura en dos bloques de información: uno relativo a las características del proceso propiamente dicho y otro relativo a los resultados e impactos. Se han seleccionado los elementos definitorios del proceso que aportan información válida para realizar estudios estadísticos a todos los niveles.

En cuanto a la definición del contenido del bloque de características nos apoyamos en la matriz del Código de Buenas Prácticas del Consejo de Europa, que permite comprender la complejidad supone un sistema de evaluación de los procesos de participación. Esta diversidad de elementos, parámetros y objetivos han sido identificadas separadamente para obtener una idea general de las posibles variables que pueden entrar en juego a la hora de evaluar un proceso.

Se establece una primera clasificación de los elementos que intervienen en la definición de un proceso según su incidencia en el mismo, se define un sistema de características:

1. Características básicas: como el tipo de iniciativa y el ámbito de actuación al que va dirigida la actividad.

2. Parámetros de partida: como el público que se pretende involucrar y el sistema de organización que podrá se formal, esto es con una estructura establecida o bien, informal que responde a un tipo de participación más ocasional y menos estructurada.

3. En cuanto al proceso se distinguen varios factores clave que se relacionan con los aspectos formales de éste, y las herramientas utilizadas, entre las que se distinguen las herramientas tradicionales y las digitales.

4. El mayor interés de esta clasificación se centra en el objetivo del proceso de participación; que a su vez se relaciona con el nivel al que se pretende participar, según la jerarquía más utilizada: información, consulta y toma de decisión.

En la configuración del BLOQUE DE CARACTERÍSTICAS se establecen tres niveles de información:

- General: en el que se recogen datos básicos que ayudan a identificar el tipo de proceso en sus condiciones básicas de partida.

- Desarrollo: en el que valora el nivel de transparencia del proceso, si se ha contado con asesoramiento técnico, los niveles de consenso a los que se ha llegado y el interés ciudadano que ha despertado dicha acción.

- Evolución: referido tanto a que la acción de participación se prolongue en el tiempo, que es lo deseable, como a los resultados a largo plazo; se evalúan los aspectos clave: como es la ejecución de los objetivos, la continuidad de mediadores o asesores técnicos en el sentido de que los expertos sean accesibles al público en general, si la actividad de participación se ha mantenido y si se han llevado a cabo evaluaciones o propuestas de mejora.

Con esta parametrización se obtienen los datos básicos que caracterizan una acción de participación en un momento concreto. Pero la participación debería ser una acción integrada en la actividad ciudadana y mantenida en el tiempo, por lo que se propone que la misma evaluación se realice en diversas etapas con el fin de conocer los procesos en su totalidad, identificar tendencias que puedan ser fomentadas o evitadas según el caso, a la mayor brevedad y prever situaciones de futuro.

La información contenida en la matriz y la valoración de los diversos parámetros, puede ser comentada y enriquecida con documentos complementarios que motiven las valoraciones, aporten otra información y consideraciones en relación al caso de estudio.

El segundo bloque de información es el de RESULTADOS, con el que se pretende analizar el impacto a nivel social de la acción realizada en los términos de los resultados del urbanismo colaborativo en los estudios de 
Innes, Boober y Healey, expuestos en el apartado anterior, que vinculan el proceso participativo con los resultados e impactos asociados a las políticas y acción pública, y los avances sociales relacionados con la construcción de consenso y el fortalecimiento de las comunidades.

En este bloque se analizan los tres aspectos considerados más relevantes relacionados con los resultados:

- Condiciones previas: se han considerado las que condicionan los procesos participativos como es la preexistencia de una cultura participativa, el contar con normas que enmarquen el sistema de participación que implica que la cultura que implica este tipo de procesos ya se está desarrollando, y en consecuencia si hay indicio para pensar que existe un sentimiento de pertenencia de la comunidad en la que se sitúa el proceso.

- Outcomes o resultado propiamente dicho: en relación a los avances sociales directos que se producen en el entorno donde se produce la colaboración, como es la generación de información sobre el proceso y sus resultados, la formación y el cambio cultural que se opera en los miembros de la comunidad en la que la participación se va asentando y la creación de redes que incluso superan el ámbito del proceso participativo.

- Impactos: entendido como avance en aspectos de calidad social, como es el respeto cívico en el que se basa el diálogo y la creación del consenso, la creación de estructuras que sostengan el proceso en el tiempo y lo fortalezcan, y por último la continuidad de la colaboración y de la cultura de la participación.

Hay que tener en cuenta que estos impactos, por ahora, son frágiles, en el sentido en que como se indica en el documento de la Agenda Urbana Española, aun no hay una cultura de participación por lo que no se ha asentado y formado parte de nuestra realidad social, se pueden producir cambios con facilidad que pueden repercutir en las condiciones de las comunidades.

\section{Ejemplo de empleo del sistema de evaluación}

Se analiza el caso de la Isla de Zorrotzaurre (La Isla Creativa), en la que los vecinos de un antiguo ámbito industrial se han organizado para frenar el Master Plan municipal, y han establecido usos alternativos en las naves abandonadas. En este caso la actividad ciudadana es reciente por lo que el análisis de resultados a largo plazo se evalúa desde el medio plazo.

\section{Elementos de análisis}

Se consideran dos etapas: una desde el inicio de la actividad vecinal en oposición al Master Plan hasta su modificación más sustancial, en 2012 y otra desde ese momento a la actualidad. Entendiendo que la actividad vecinal no está tan centrada en la definición del Master Plan, cuyo desarrollo está bastante ralentizado. Y que han entrado nuevos grupos con peso en la actividad cívica como son los responsables de las actividades que se desarrollan en los edificios existentes.

Respecto a la situación inicial se parte de un grupo vecinal estructurado pero no organizado, ni con experiencia en cuestiones de participación, si bien la iniciativa surge como irrupción, dentro del denominado urbanismo radical.

El aprendizaje practicando, basado en la implicación personal de determinados participantes, y el conocimiento técnico producen los resultados esperados o al menos cambiar el plan en las cuestiones más fundamentales.

En la actualidad los grupos y asociaciones implicadas son muy numerosos, se han creado redes fuertes y estructuradas. Aunque no parece que haya una organización que coordine a estos grupos, o que genere procesos de participación organizados como tal. 


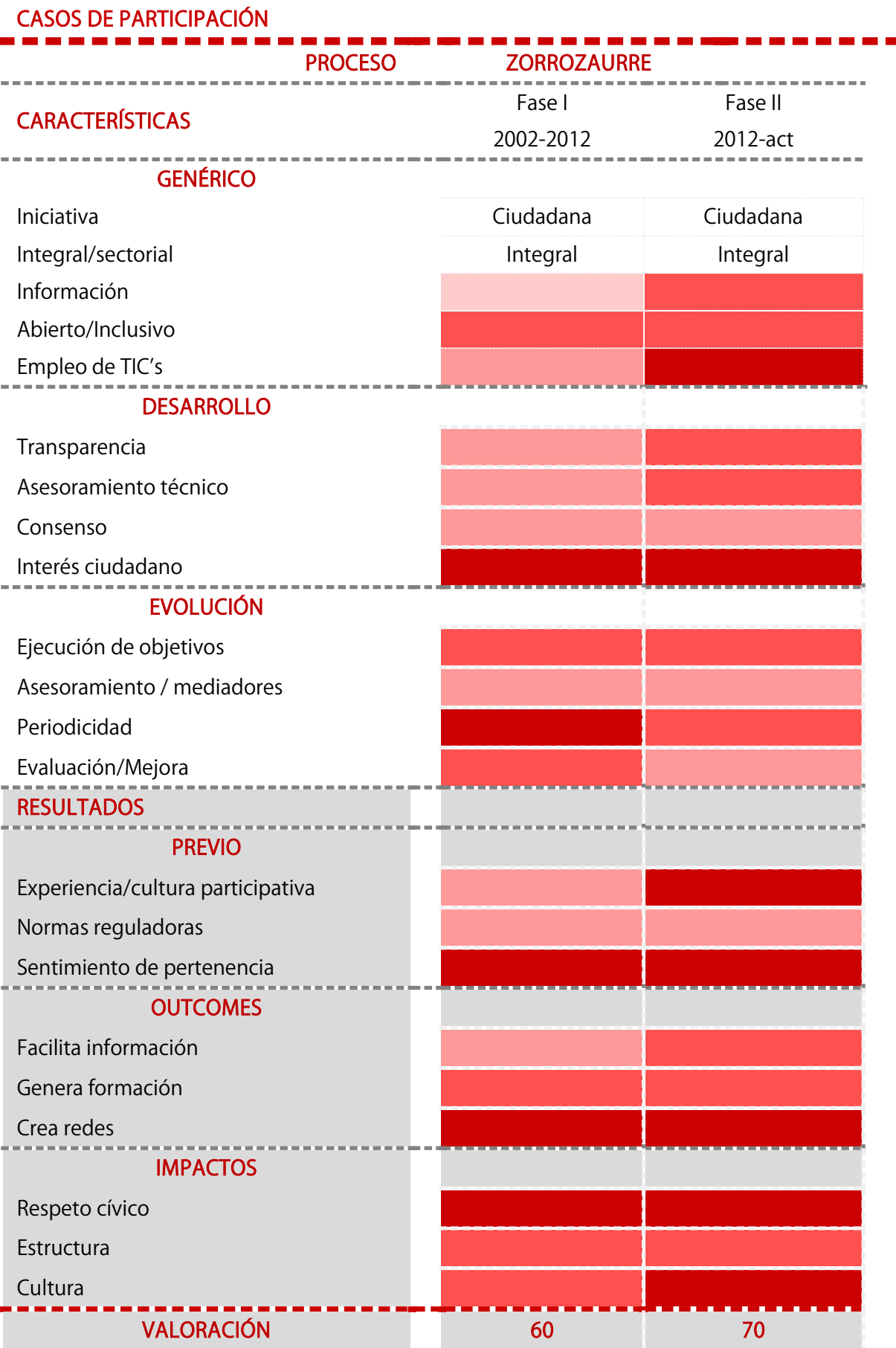

Tabla 4. Evaluación del proceso de participación de Zorrozaurre. Fuente: Elaboración propia

\section{Evaluación}

Se echa de menos la existencia de un organismo que coordine las relaciones entre administración -o comisión gestora- $y$ asociaciones y vecinos de la isla, formado por representantes de ambos, que facilite la creación de un marco de actuación y de toma de decisiones; si bien existen negociaciones parece que hay una falta de diálogo y de normas que estructuren dicha relación. Llama la atención esta situación teniendo en cuenta la actividad municipal en cuestiones de participación. 
La información disponible está sectorizada, información de la comisión gestora y por otro lado información de los diversos grupos. En cuanto a la información de los vecinos parece que ha perdido vitalidad y se encuentra desactualizada. Esta diversidad de fuentes manifiesta la falta de acuerdo y por tanto que aún no se ha llegado a una situación consenso y colaboración.

\section{BIBLIOGRAFÍA}

Obras completas

AEVAL (2010). Fundamentos de evaluación de políticas públicas. Madrid: Ministerio de Política Territorial y Adm. Pública. Agencia Estatal de Evaluación de las Políticas Públicas y la Calidad de los Servicios.

ALEXANDER, Ch. (1975). Urbanismo y participación: el caso de la Universidad de Oregón. (Edición en castellano de 1976). Barcelona: Gustavo Gili (Título original: The Oregon experiment).

ECTP-CEU. (2015). La Carta Europea de la Democracia Participativa en procesos urbanísticos. Dublín.

AUE (2019) Agenda Urbana Española. Madrid: Ministerio de Fomento.

FARIÑA, J. y NAREDO, JM (Dir) (2010). Libro Blanco de la Sostenibilidad en el Planeamiento Urbanístico Español. Madrid: Ministerio de Vivienda.

FERNÁNDEZ, M. y GIFREU, J. (2016). El Uso temporal de los vacíos urbanos. Serie Urbanismo y Vivienda Diputación de Barcelona: Colección Estudios.

GREGORIO, S. de (2012). Políticas Urbanas de la Unión Europea desde la perspectiva de la planificación colaborativa. Las iniciativas Comunitarias URBAN y URBAN II en España. Madrid: Universidad Politécnica de Madrid.

HEALEY, P. (2006-1997). Collaborative Planing: Shaping Places in fragmentes Societies. Londres: Macmillan (ed. Origin, Londres 1997)

INNES, J. Y BOOHER, D. (2000). Public Participation in Planning: New Strategies for the 21st Century. Berkely: Institute of Urban and Regional Development, University of California.

JACOBS, J. (1961) Muerte y Vida de las grandes ciudades. Madrid: Capitán Swing. 3a edición 2013.

LEFEBVRE, H. (1968). El derecho a la ciudad. (Edición en castellano de 1969). Barcelona: Ediciones 62. (Título original: Le droit à la ville).

MOORE, M. (1998). Gestión estratégica y creación de valor en el sector público. Barcelona: Paidós.

OCDE (2006). Participación Ciudadana. Manual de la OCDE sobre la información, consulta y participación en la elaboración de políticas públicas.

PAREJO, L. (2016). Urbanismo temporal, derecho a la ciudad y marco estatal de las políticas urbanas. En El Uso temporal de los vacíos urbanos. Manuel Fernández y Judith Gifreu. Serie Urbanismo y Vivienda. Diputación de Barcelona: Colección Estudios.

SUBIRATS, J. (2001). Nuevos mecanismos participativos y democracia: promesas y amenazas, en Ciudadanos y decisiones públicas, J.Font (ed) Barcelona: Ariel. P33-42

TORRAS, L. (2016). El espacio del técnico municipal: el Pla BUITS como síntoma. En El Uso temporal de los vacíos urbanos. Manuel Fernández y Judith Gifreu. Serie Urbanismo y Vivienda. Diputación de Barcelona: Colección Estudios.

Revistas

ALGUACIL, J. (2000). Ciudad, ciudadanía y Democracia urbana. Documentación Social, No119. 
CAZ, R. del y SARABIA, M. (2005). ¿Participar o ser participado? 200 preguntas sobre participación en el urbanismo en Participación ciudadana para el urbanismo del siglo XXI. Valencia: ICARO,CTAV.

CONSEJO DE EUROPA (2009). Código de Buenas Prácticas para la Participación Ciudadana en el proceso de toma de decisiones. En Conferencia de OING. Estrasburgo: Consejo de Europa 1-19.

CONSEJO EUROPEO DE URBANISTAS.(2003). Nueva Carta de Atenas 2003. La Visión de las ciudades en el siglo XXI del Consejo Europeo de Urbanistas. Parte B.

DAVIDOFF, P. (1965). Advocacy and pluralism in Planning. En Journal oj the American Institute of Planning $\mathrm{n}^{\circ}$ 31, pp. 331-338.

FERNÁNDEZ, B. (2013). Políticas urbanas innovadoras, gobernanza y planificación flexible. Análisis de la evolución en Francia y propuestas de adaptación al contexto español. En Estudios de Progreso n072. Fundación Alternativas.

FORESTER, J. (2000). The Deliberative Practitioner: Encouraging participatory planning processes. Cambridge (Mass.) y Londres: The MIT Press.

GARCÍA-BELLIDO, J. (1978). Participación popular y lucha de clases en el planeamiento urbanístico. Esbozos para un debate, CAU: construcción, arquitectura, urbanismo, № 51.

INNES, J. (1995). Planning theory's emergining paradigm: Communicative action and interactive practice. En Journal of Planning Education and Research ,Volumen 14 (3), pp. 128-135.

MORENO-BALBOA, C. (2017) Urbanismo Colaborativo. CIUR, $n^{\circ} 115$

SECRETARÍA TÉCNICA DEL OLDP, (2006) Guía práctica Evaluación de procesos participativos. Ajuntament de Barcelona

VERDAGUER, C. (2003). Por un urbanismo de los ciudadanos en Ecología y Ciudad. Raíces de nuestros males y modos de tratarlos. Madrid: Fundación de Investigaciones Marxistas. Ediciones de Intervención Cultural. El Viejo Topo.

\section{Fuentes electrónicas}

AEVAL (2010) http://www.aeval.es/es/index.html (Consultado: 4/04/2017)

https://elblogdefarina.blogspot.com/2017/11/once-plazas-de-madrid-1.html (Consulta: 8/03/2019)

http://ajuntament.barcelona.cat/ecologiaurbana/es/que-hacemos-y-porque/ciudad-verde-y-biodiversidad/plabuits (Consulado: 14/05/2017)

http://www.undp.org/content/undp/es/home/ourperspective/ourperspectivearticles/2013/03/19/sustainabledevelopment-goals-and-the-post-2015-agenda-why-does-participation-matter-.html. (Consultado: 15/03/2017)

Mapa Los Madriles: http://www.viveroiniciativasciudadanas.net/civics/iniciativas/ (Consulta: 8/03/2018) 